\title{
GSM based Wireless Sensor Network to Measure Global Warming, Humidity and $\mathrm{CO} 2$
}

\author{
Md.Khaled Hossain \\ Graduate member, IEEE \\ A.B.A Nir Nirzan, Plot\#2515, \\ H\# G3, Ashkona, Dhaka.
}

\author{
S.M.Ariful Haque \\ Lecturer, UITS \\ Jamalpur, twin tower, Baridhara \\ ga-37/1, Block-j, Dhaka.
}

\author{
Sumit Bhattacharyya \\ Lecturer, ADBPB \\ House no\# 05, Avenue\# 03, \\ Mirpur-02, Hazi Road, Dhaka.
}

\begin{abstract}
This paper discusses GSM based global wireless sensor network to measure increase of global warming \& air pollution. Each motes of the wireless sensor network can cover the area wherever GSM network is available. Each mote consists of temperature, humidity \& co2 measuring sensors, equipped with low power consuming GSM module, data logging facility \& real time stamp. Motes can communicate with each other but most of the time they communicate with the central receiver. Central receiver is capable of receiving calls from modes, display the data in portable TFT LCD screen \& also transmit data to computer through USB. To transmit data sensors generate call to receive station. To receive data receiver first verify the caller ID and then it receives the call. Sensors send or receive data through Dual tone multi frequency (DTMF) and will receive commands through DTMF also. Lots of control system has been designed using DTMF but in this project DTMF tones are used to create all characters of an ASCII table. The number of motes can be connected are unlimited and the coverage area is also unlimited in this project.
\end{abstract}

\section{General Terms}

Wireless Sensor Network, GSM, DTMF, Sensors.

\section{INTRODUCTION}

Wireless Sensor networks have gained tremendous attention in present research fields. It has unlimited application and significance in environment [1] [2], $\mathrm{CO}_{2}$ monitoring [3], agriculture [4], Habitat monitoring [5]. But all these wireless sensor networks are for limited number of motes and limited coverage area. To cover a big area network need complex routing and large expanses. To overcome this obstacle GSM based wireless sensor network have been designed. In this network sensors will communicate through dual tone multi frequency (DTMF). So far DTMF has been used as commanding tones only with limited signals. There are few research papers where DTMF is used as communication medium in wireless sensor network [6]. Yun Chan Cho and Jae wook Jeon [7] used DTMF of mobile phone. D. Manojkumar et.al. [8] Controlled a robot by a mobile using DTMF tone. To control domestic systems DTMF has been used [9]. Smart phones also have been used to control mobile robots [10]. Human-Robot interaction mechanisms that allow a human commander to control a mobile robot via cellular phone have been developed and successfully tested by Ali Sekmen et.al. [11] and T. Kubik et.al. [12]. Tho Nguyen and Linda G. Bushnell have implemented DTMF communication For Robots using DTMF transceiver [13]. But all of them are used as limited controlling signal. Only 9 buttons has been used just to control relay, cars or communicate etc. In our project using DTMF tone complete ASCII chart have been implemented.

Communication between motes and receiver can be initiated from any one anytime. Motes store sensors data including time stamp in SD memory card. To reduce the cost of communication motes generate call during off peak hour after 12am. Receiver GSM module will remain in auto receive mode. After receiving the call, motes start sending DTMF tones of encoded sensor data. Each mote has GSM modem as DTMF transceiver. From transmitting side the ASCII value of information will be encoded and transmit through DTMF tone. Receiver side will receive DTMF tone and decode it into ASCII value. These decoded data will be fetching to computer through USB. Matlab receives the data and shows it in GUI.

\section{WSN DESCRIPTION}

To Develop the WSN each motes are connected with receiver through GSM network. As GSM network is already established worldwide this is the only way to establish a WSN to measure global warming, humidity \& $\mathrm{CO}_{2}$. Each mote consists of sensors, real time clock module, 2 GB SD card, GSM module \& atmega8 microcontroller. 3 sensors are used in each mote, temperature sensor, humidity sensor $\& \mathrm{CO}_{2}$ gas sensor. Real time clock is used to have exact time stamp of each measurement. The RTC module provides time \& day, month, year calculations. SD card is used to store the sensor values locally. It might happen that mote does not have enough power to transmit the signal but still motes are capable of logging data in SD cards and data can be collected from SD card manually. To transmit sensors values each mote also has GSM modem. The modem only generates calls after $12 \mathrm{am}$ at off peak hour to save the call charge. And heart of each mote is atmega 8 microcontroller. It is a small size micro-controller and consumes small amount of power while running in sleep mode. This whole WSN runs on interrupt driven system. After a fixed amount of time interval micro-controller wakes up and collect data from sensors, collect real time stamp, stores data into SD card and then again goes back to sleep. When the time comes to transmit data it wakes up, generate call to receive terminal and transmit all stored data within around 3 to 4 minutes. This system runs on battery and GSM modem consumes lots of power. To longer the lifetime of each mote GSM modules is turned on only once each day. Receive terminal is connected with computer. Though this project is focused on global warming but we were unable to place motes in different countries. This project has implemented within Dhaka city with two motes and one receiver. 


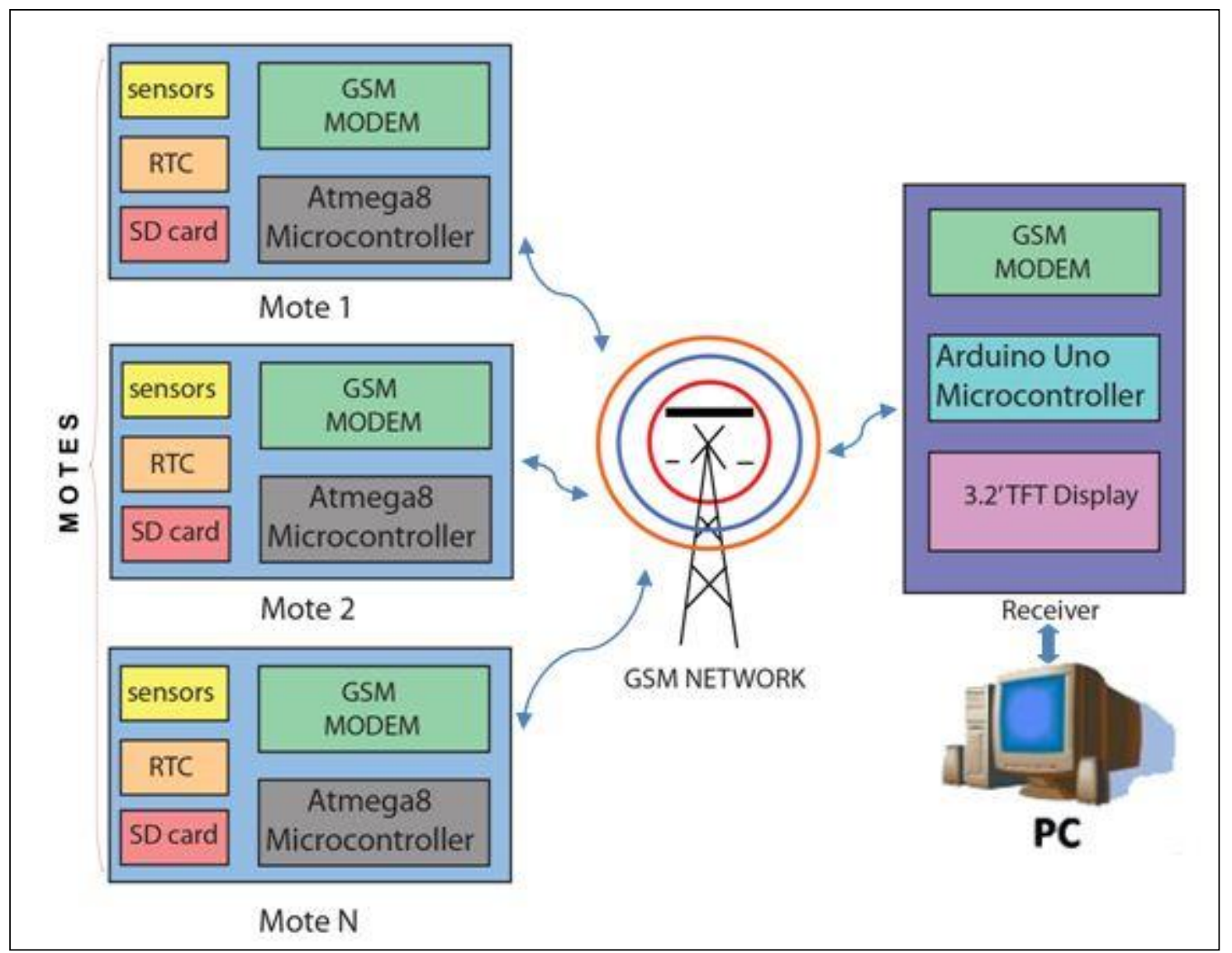

Fig 1: GSM based WSN environment monitoring system

\section{HARDWARE DESIGN}

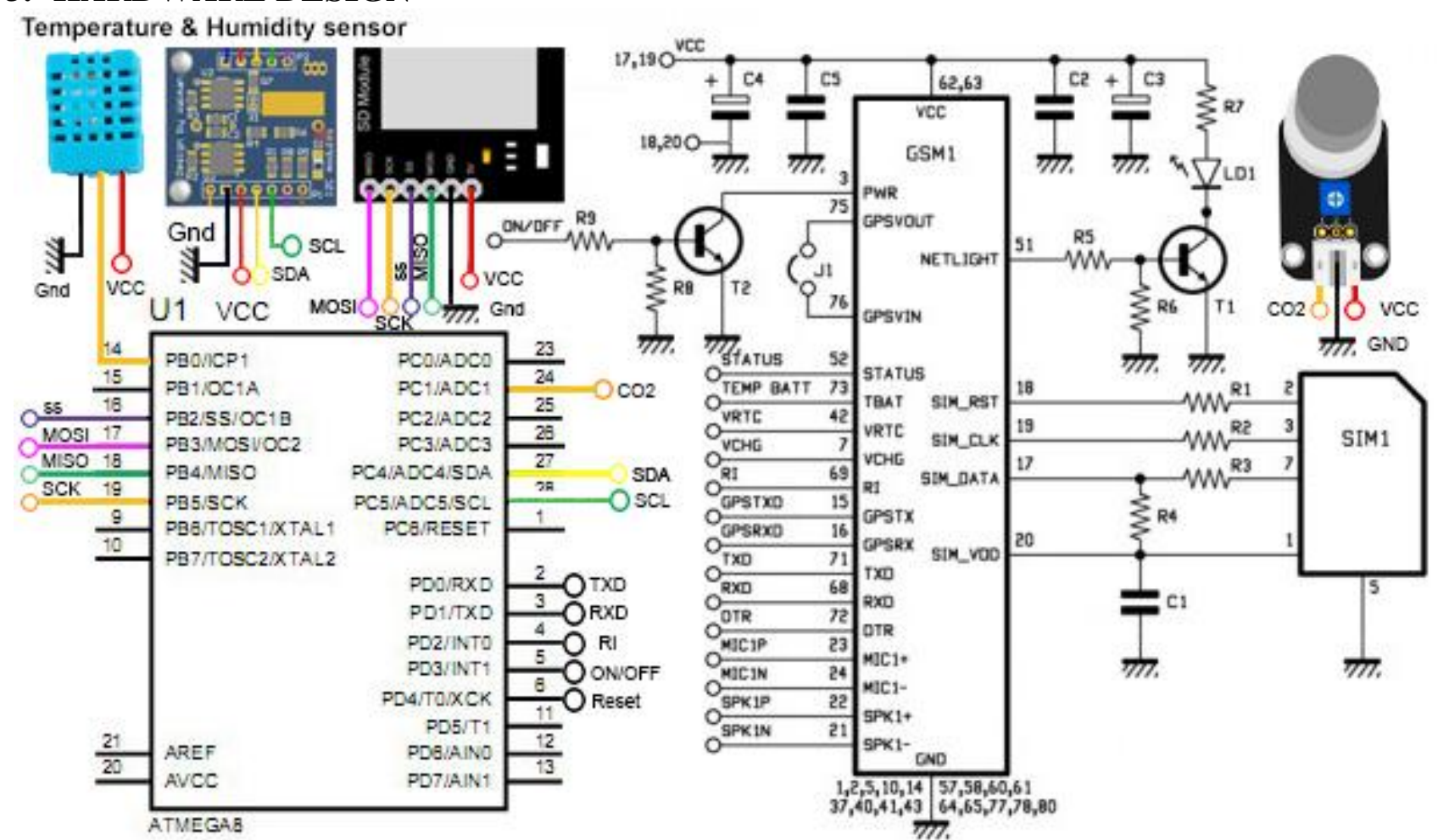

Fig2:- Schematic Diagram of a single Mote 
Heart of each mote is atmega8. Temperature and humidity sensor has 3pins; sensor gives digital output which is connected to PB0 pin. I2C RTC module (Real Time Clock) is connected to pin $27 \& 28$. SD card module has SPI (Serial Peripheral Interface). MISO, MOSI, SCK \& SS pin of SD card module is connected with $16,17,18,19$ pins of atmega8. GSM module consumes lots of power that's why the on off pin of GSM module is controlled by atmega8 PD3 pin. GSM module communicates with atmeg8 through UART. RXD of GSM module is connected with TXD of atmega8 and TXD of GSM module is connected to RXD of atmega8. $\mathrm{CO}_{2}$ sensor communicates through $\mathrm{PC} 1$ pin which is an ADC pin.

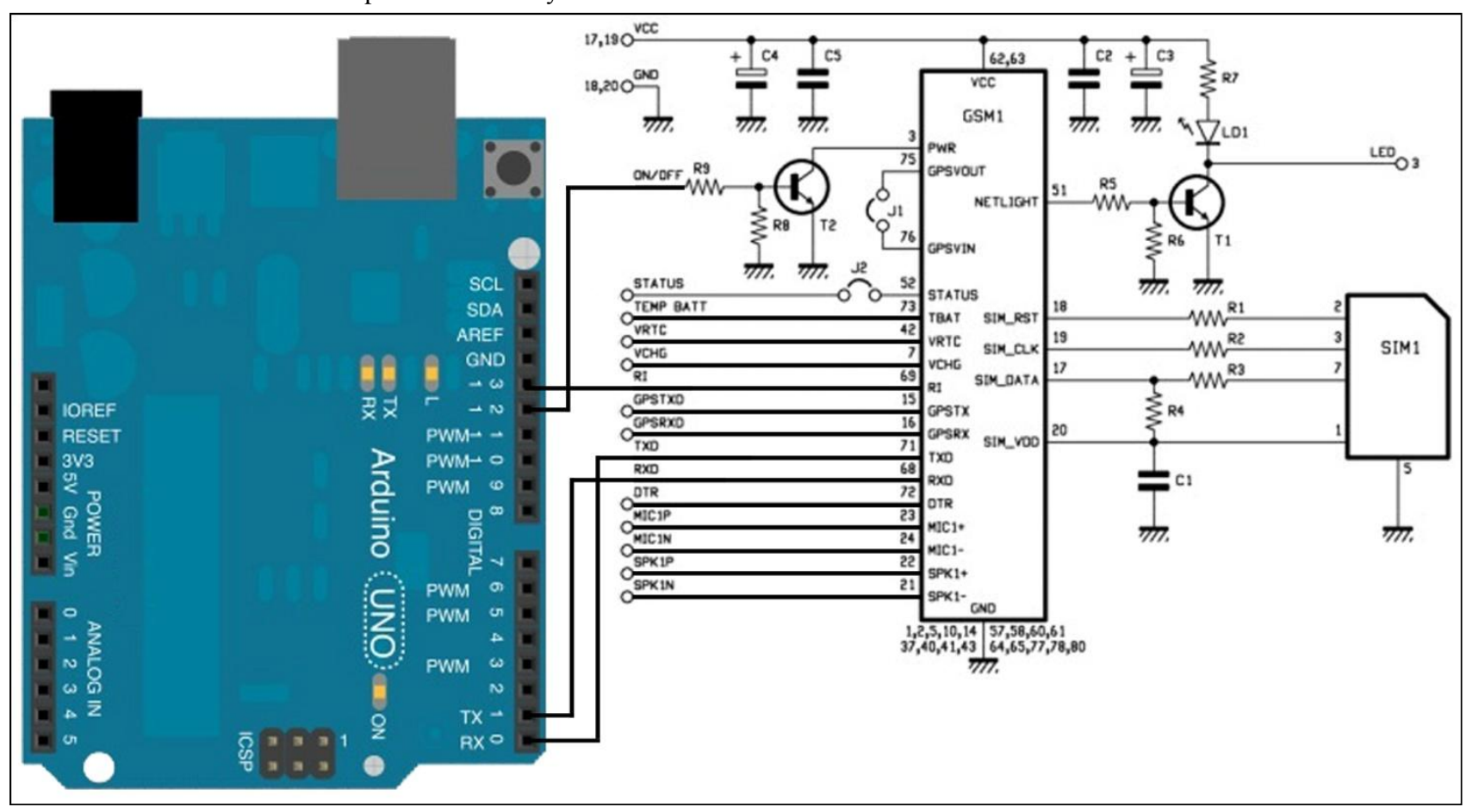

Fig3: Schematic of receiver module

In receiver circuit arduino is used as microcontroller. With ardruino GSM module is interfaced. Ardruino is connected with computer through USB port. But communication protocol is UART and baud rate is 9600 .

\section{WSN DEVELOPMENT}

Sensors and other equipments have been chosen specifically for wireless sensor network. Most of the devices consume less power in sleep mode. Here descriptions of equipments are provided bellow.

\subsection{Sensors}

There are two sensors has been used in this project. One is temperature, humidity sensor and another is $\mathrm{CO}_{2}$ sensor. There are lots of sensors available in market but less power consuming sensors has been selected.

\subsubsection{DHT11 temperature-humidity sensor}

The DHT11 is low-cost digital temperature and humidity sensor. It uses a capacitive humidity sensor and a thermostat to measure the surrounding air, and output digital values of temperature and humidity to ardruino.
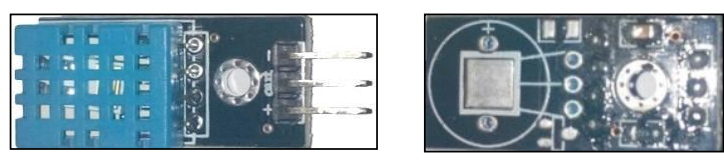

Fig4: Temperature $\&$ humidity sensor front $\&$ rear view

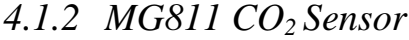

The MG811 is a inexpensive CO2 sensor, Good sensitive co2 sensor. It can detect $\operatorname{co} 2$ with good accuracy.
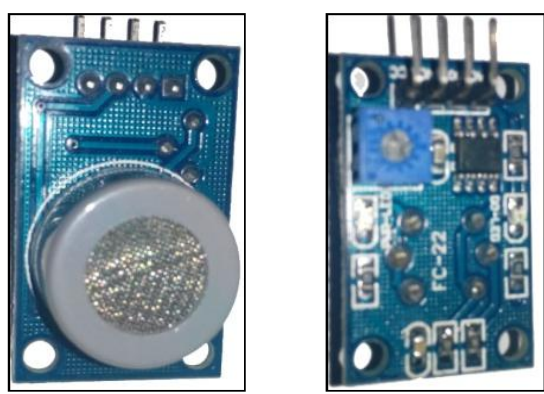

Fig5: CO2 gas sensor front $\&$ rear view

MG811 has good sensitivity and selectivity to $\mathrm{CO} 2$ \& low humidity and temperature dependency. It has long stability which makes it suitable for rough environment. Its ranges are given bellow.

- Detection Range: 0 - 10,000 PPM CO2

- Response Time: $<60 \mathrm{~s}$

- Heater Voltage: $6.0 \mathrm{~V}$ 


\subsection{RTC module}

This module is With LIR2032 rechargeable lithium battery. About 1 year run time with full BCD clock calendar chip of 56byte non-volatile RAM. The chip can provide second, minute, hour etc information. Built-in power sensor circuit in the chip, with brownout detection and battery switch function. Under battery backup mode, power consumption is below $500 \mathrm{uA}$. It provides accurate calendar up to year 2100 [14].
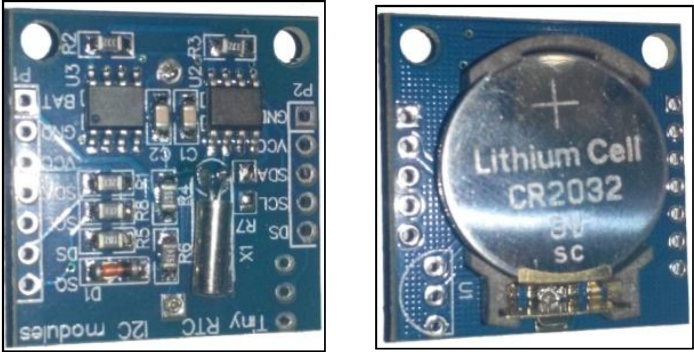

Fig6: I2C RTC module

\subsection{SD card module}

SD card module is interfaced with microcontroller through SPI interface. A 4GB SD card is used to log data for one year. Here is the picture of SD card module.

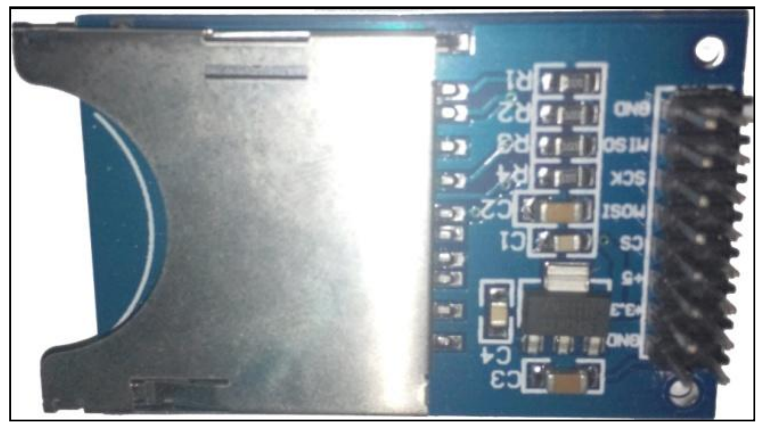

Fig7: SPI SD card module

\subsection{GSM module}

Sim908 has been used as GSM module. SIM908 module is a complete Quad-Band GSM/GPRS module which combines GPS technology for satellite navigation. The compact design which integrated GPRS and GPS in a SMT package will significantly save both time and costs. Power consumption (GSM engine in idle mode) around $77 \mathrm{~mA}$.

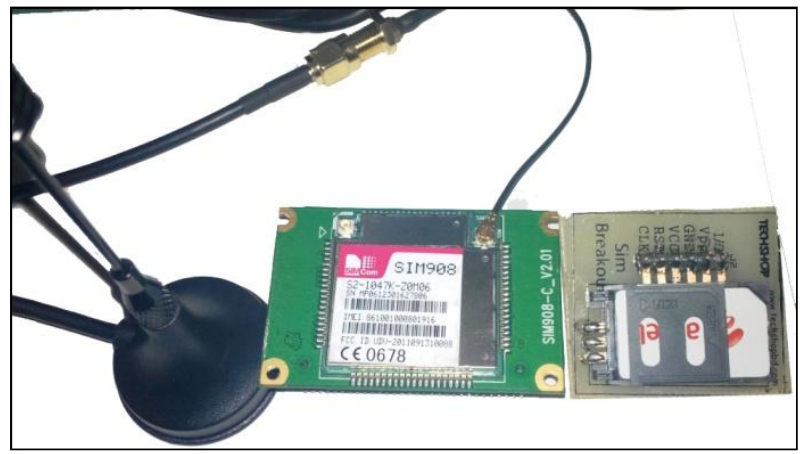

Fig8: SIM908 GSM module

\subsection{Atmega8 Microcontroller}

Brain of this project is Atmega8 micro-controller. It is a 8 bit Micro controller with RISC architecture. Its speed is up to 16MIPS throughput at $16 \mathrm{MHz}$. It has $8 \mathrm{~K}$ bytes of flash and 512 bytes EEPROM. Operating voltage $2.7 \mathrm{v}-5.5 \mathrm{v}$, in active mode it consumes only $3.6 \mathrm{~mA} \&$ in sleep mode it consumes less than 1uA current [15] which made it a perfect choice for this project.

\subsection{Arduino Uno}

The Arduino Uno is a microcontroller board based on the ATmega328. It has 14 digital input/output pins, 6 analog inputs, a $16 \mathrm{MHz}$ ceramic resonator, a USB connection. The Uno differs from all preceding boards in that it does not use the FTDI USB-to-serial driver chip. Instead, it features the Atmega16U2 (Atmega8U2 up to version R2) programmed as a USB-to-serial converter [16].

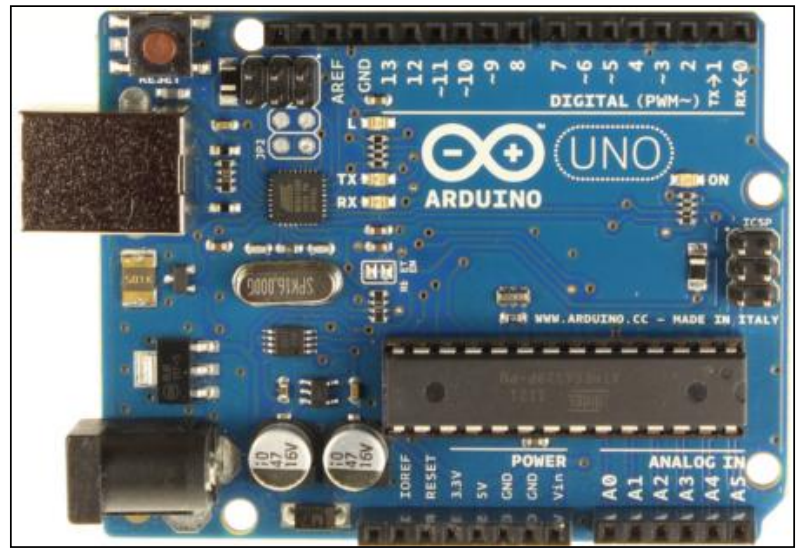

Fig9: Arduino Uno front side

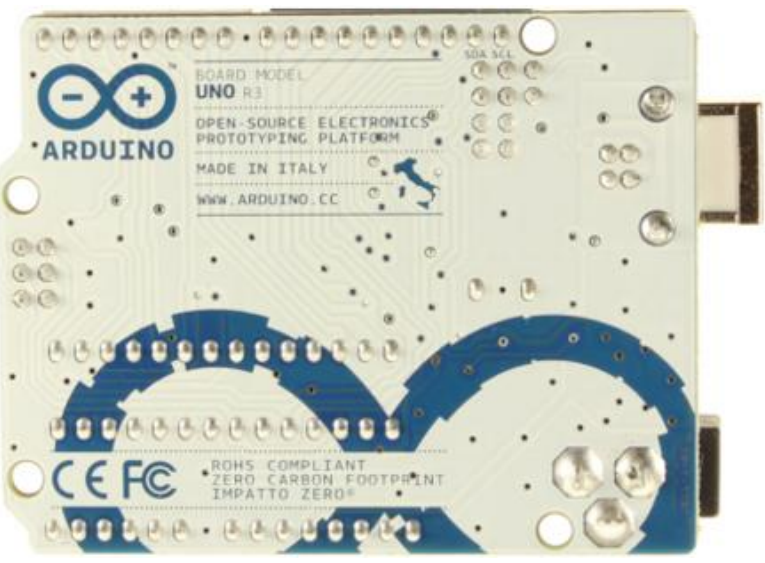

Fig10: Arduino Uno rear side.

Arduino Uno has built in USB to sear converter. It makes the task easy to communicate with PC. It can transmit data to Matlab and also able to receive commands from Matlab. Communication protocol is UART. The baud rate of serial communication is 9600. Arduino Uno is capable of communicate in higher baud rate but this is the standard and most stable baud rate for data communication as lots of data don't need to be transferred. In this project serial port3 is used for data transfer. 


\section{MAINT TECHNOLOGY USED}

\subsection{DTMF Tone}

DTMF generation is a composite sinusoidal signal of two tones between the frequency of $697 \mathrm{~Hz}$ and $1633 \mathrm{~Hz}$ [11]. The DTMF keypad is arranged such that each row will have its own unique tone frequency and also each column will have its own unique tone. Below is a representation of the typical DTMF keypad and the associated row/column frequencies.

\section{HIGH GROUP TONES}

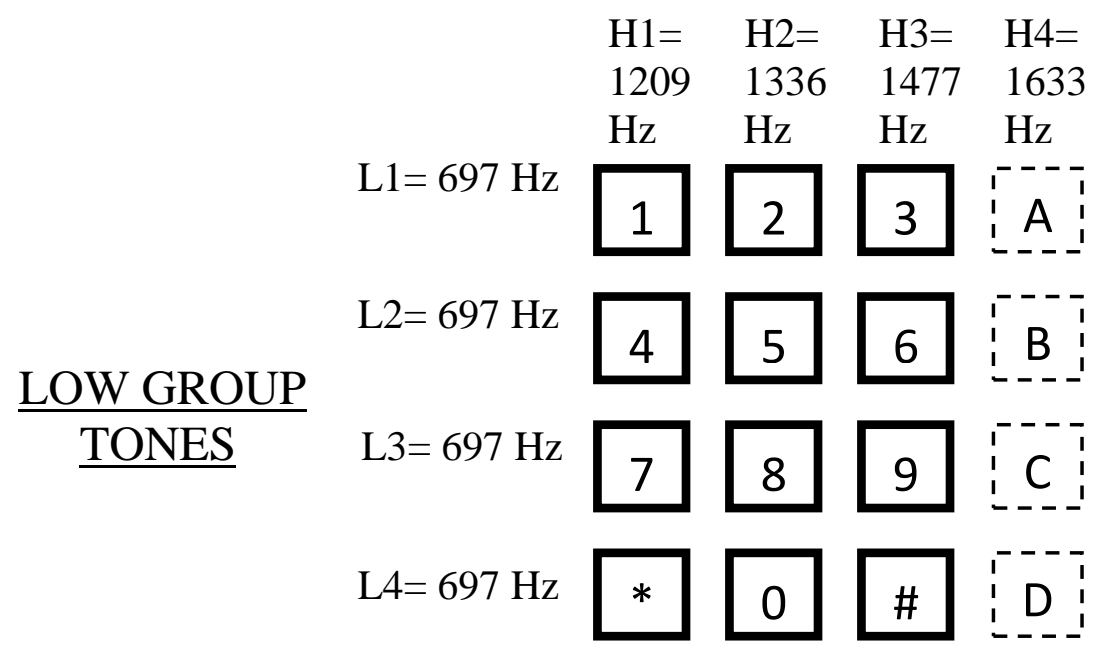

Fig 11:- DTMF keypad layout.

Symbol *1* [697 1209]

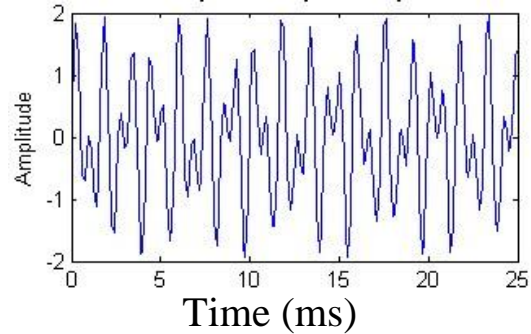

Symbol *2* [697 1336]

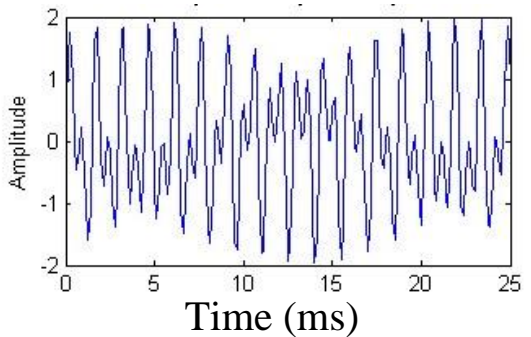

Symbol *3* [697 1477]

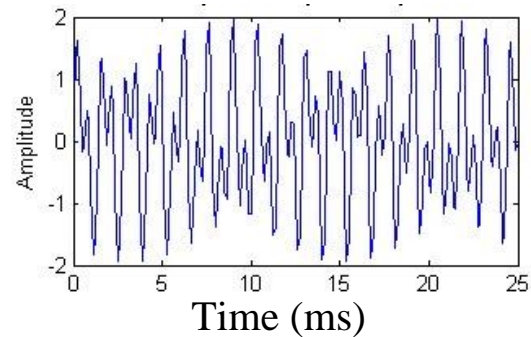

Figure 12:- DTMF frequency when one digit is pressed

\subsection{Software Defined DTMF:-}

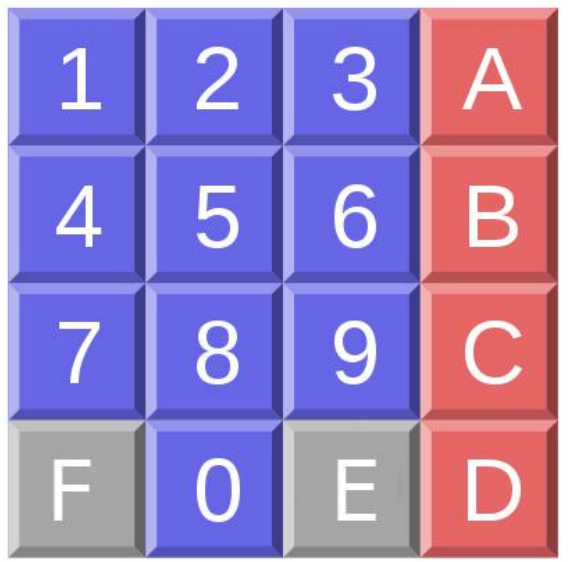

Fig 13:- DTMF keypad with digits E, F added

In this project $* \&$ \# button has been eliminated and added $\mathrm{E} \& \mathrm{~F}$ button. Now this keypad contains all 16 digits of BCD (binary coded decimal) digits. Table value of DTMF tones has been changed according to $\mathrm{BCD}$ values. Bellow is the chart of values of all 16 digits.

Table II: - Software defined DTMF tone values

\begin{tabular}{|c|c|c|c|c|c|}
\hline KEY & TOE & Q4 & Q3 & Q2 & Q1 \\
\hline 0 & 1 & 0 & 0 & 0 & 0 \\
\hline 1 & 1 & 0 & 0 & 0 & 1 \\
\hline 2 & 1 & 0 & 0 & 1 & 0 \\
\hline 3 & 1 & 0 & 0 & 1 & 1 \\
\hline 4 & 1 & 0 & 1 & 0 & 0 \\
\hline 5 & 1 & 0 & 1 & 0 & 1 \\
\hline 6 & 1 & 0 & 1 & 1 & 0 \\
\hline 7 & 1 & 0 & 1 & 1 & 1 \\
\hline 8 & 1 & 1 & 0 & 0 & 0 \\
\hline 9 & 1 & 1 & 0 & 0 & 1 \\
\hline A & 1 & 1 & 0 & 1 & 0 \\
\hline B & 1 & 1 & 0 & 1 & 1 \\
\hline
\end{tabular}




\begin{tabular}{|c|c|c|c|c|c|}
\hline $\mathrm{C}$ & 1 & 1 & 1 & 0 & 0 \\
\hline $\mathrm{D}$ & 1 & 1 & 1 & 0 & 1 \\
\hline $\mathrm{E}$ & 1 & 1 & 1 & 1 & 0 \\
\hline $\mathrm{F}$ & 1 & 1 & 1 & 1 & 1 \\
\hline ANY & 0 & $\mathrm{Z}$ & $\mathrm{Z}$ & $\mathrm{Z}$ & $\mathrm{Z}$ \\
\hline
\end{tabular}

form an ASCII character we need two BCD digits. To represent any digit robot1 has to send two DTMF tones. For Example if robotl wants to send a character ' $\mathrm{H}$ ' it will send DTMF tones $4 \& 8$ as the hex value of ' $H$ ' is $0 x 48$. In this way robots can send any character it wants from ASCII table. Bellow the ASCII chart is shown and corresponding DTMF tones in red color.

Microcontroller receives original DTMF value but it converts the original value into the above value using look up table. To

Table III: - Complete ASCII chart using DTMF tones only

\begin{tabular}{|c|c|c|c|c|c|c|c|c|c|c|c|c|c|c|c|c|}
\hline & 0 & 1 & 2 & 3 & 4 & 5 & 6 & 7 & 8 & 9 & $\mathrm{~A}$ & B & $\mathrm{C}$ & $\mathrm{D}$ & $\mathrm{E}$ & $\mathrm{F}$ \\
\hline 0 & $\begin{array}{l}\text { NUL } \\
\text { 0x00 }\end{array}$ & $\begin{array}{l}\text { SOH } \\
\text { Ox01 }\end{array}$ & STX & ETX & EOT & ENQ & ACK & BEL & BS & HT & LF & $\mathrm{VT}$ & $\mathrm{FF}$ & CR & SO & $\begin{array}{c}\text { SI } \\
\text { 0x0F }\end{array}$ \\
\hline 1 & DLE & DC1 & DC2 & DC3 & DC4 & NAK & SYN & ETB & CAN & EM & SUB & ESC & FS & HS & RS & US \\
\hline 2 & SPC & $!$ & "6 & \# & $\$$ & $\%$ & $\&$ & ' & ( & ) & $*$ & + & 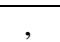 & - & . & I \\
\hline 3 & 0 & 1 & 2 & 3 & 4 & 5 & 6 & 7 & 8 & 9 & : & ; & $<$ & $=$ & $>$ & $?$ \\
\hline 4 & $@$ & A & B & C & D & $\mathrm{E}$ & $\mathrm{F}$ & $\mathrm{G}$ & $\mathrm{H}$ & I & $\mathrm{J}$ & K & $\mathrm{L}$ & M & $\mathrm{N}$ & $\mathrm{O}$ \\
\hline 5 & $\mathrm{P}$ & $\mathrm{Q}$ & $\mathrm{R}$ & $\mathrm{S}$ & $\mathrm{T}$ & $\bar{U}$ & $\mathrm{~V}$ & $\mathrm{~W}$ & $\mathrm{X}$ & $\mathrm{Y}$ & $\mathrm{Z}$ & {[} & 1 & ] & $\wedge$ & _ \\
\hline 6 & ᄃ & $\mathrm{a}$ & $\mathrm{b}$ & $\mathrm{c}$ & $\mathrm{d}$ & $\mathrm{e}$ & $\mathrm{f}$ & $\mathrm{g}$ & $\mathrm{h}$ & $\mathrm{i}$ & $\mathrm{j}$ & $\mathrm{k}$ & 1 & $\mathrm{~m}$ & $\mathrm{n}$ & $\mathrm{o}$ \\
\hline 7 & $\mathrm{P}$ & $q$ & $\mathrm{r}$ & $\mathrm{s}$ & $\mathrm{t}$ & $\mathrm{u}$ & $\mathrm{V}$ & $\mathrm{W}$ & $\mathrm{x}$ & $\mathrm{y}$ & $\mathrm{Z}$ & \{ & 1 & \} & $\sim$ & DEL \\
\hline 8 & $€$ & & 6 & $f$ & , & $\ldots$ & $\dagger$ & $t$ & ^ & $\% 0$ & Š & $<$ & $\mathrm{E}$ & & Ž & \\
\hline 9 & & 6 & , & " & $"$ & - & - & - & $\sim$ & TM & $\check{S}$ & $>$ & $œ$ & & $\bar{Z}$ & $\ddot{Y}$ \\
\hline $\mathrm{A}$ & & $\mathrm{j}$ & $\phi$ & $£$ & 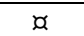 & $¥$ & 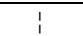 & $\S$ & 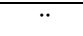 & (C) & $\mathrm{a}$ & $\ll$ & ᄀ & - & (B) & - \\
\hline B & $\circ$ & \pm & 2 & 3 & ' & $\mu$ & II & . & & 1 & 0 & $\gg$ & $1 / 4$ & $1 / 2$ & $3 / 4$ & i \\
\hline C & $\grave{A}$ & Á & $\hat{\mathrm{A}}$ & $\tilde{\mathrm{A}}$ & $\ddot{\mathrm{A}}$ & $\AA$ & Æ & Ç & $\overline{\mathrm{E}}$ & É & $\hat{\mathrm{E}}$ & $\ddot{\mathrm{E}}$ & $\grave{I}$ & Í & $\hat{\mathrm{I}}$ & $\ddot{\mathrm{I}}$ \\
\hline $\mathrm{D}$ & $\mathrm{D}$ & $\tilde{\mathrm{N}}$ & Ò & Ó & $\hat{\mathrm{O}}$ & $\tilde{O}$ & $\ddot{O}$ & $x$ & $\varnothing$ & $\overline{\mathrm{U}}$ & Ú & $\widehat{\hat{U}}$ & $\ddot{\mathrm{U}}$ & $\dot{Y}$ & $\mathrm{P}$ & $\beta$ \\
\hline $\mathrm{E}$ & $\grave{A}$ & á & $\hat{\mathrm{a}}$ & $\tilde{\mathrm{a}}$ & $\ddot{a}$ & $\bar{a}$ & æ & ç & è & é & $\hat{\mathrm{e}}$ & $\ddot{\mathrm{e}}$ & ì & í & $\hat{\imath}$ & $\ddot{1}$ \\
\hline $\mathrm{F}$ & $\begin{array}{c}\text { Ð } \\
\text { 0xF0 }\end{array}$ & $\tilde{\mathrm{n}}$ & ò & ó & ô & $\tilde{\mathrm{o}}$ & $\ddot{0}$ & $\div$ & $\varnothing$ & ù & ú & $\hat{\mathrm{u}}$ & $\ddot{\mathrm{u}}$ & ý & $\mathrm{p}$ & $\begin{array}{c}\ddot{Y} \\
0 x F F\end{array}$ \\
\hline
\end{tabular}

\section{DATA LOGGING:-}

Each sensor sends data to receiver and receiver transmit it to computer. Here data logging of temperature and humidity is shown in serial terminal.

\begin{tabular}{|lll||} 
Humidity: $32.70 *$ & Temperature: $25.70 * \mathrm{C}$ \\
Humidity: $32.70 *$ & Temperature: $25.70 * \mathrm{C}$ \\
Humidity: $32.70 *$ & Temperature: $25.70 * \mathrm{C}$ \\
Humidity: $32.70 *$ & Temperature: $25.70 * \mathrm{C}$ \\
Humidity: $32.60 *$ & Temperature: $25.70 * \mathrm{C}$ \\
Humidity: $32.60 *$ & Temperature: $25.70 * \mathrm{C}$ \\
Humidity: $32.60 *$ & Temperature: $25.70 * \mathrm{C}$ \\
Humidity: $32.60 *$ & Temperature: $25.70 * \mathrm{C}$ \\
Humidity: $32.60 *$ & Temperature: $25.40 * \mathrm{C}$ \\
Humidity: $32.60 *$ & Temperature: $25.40 * \mathrm{C}$ \\
Humidity: $32.60 *$ & Temperature: $25.40 * \mathrm{C}$ \\
Humidity: $32.60 *$ & Temperature: $25.40 * \mathrm{C}$ \\
Humidity: $32.60 *$ & Temperature: $25.90 * \mathrm{C}$ \\
Humidity: $32.60 *$ & Temperature: $25.90 * \mathrm{C}$ \\
Humidity: $32.60 *$ & Temperature: $25.90 * \mathrm{C}$ \\
Humidity: $32.60 *$ & Temperature: $25.90 * \mathrm{C}$ \\
\hline$\square$ Autoscroll & & \\
\hline
\end{tabular}

Fig14: logging data of temperature \& humidity 


\section{FURTHER APPLICATION}

a. Robots community development and share intelligence.

b. GPRS based network using internet.

c. Wireless Robot Control.

d. Industry and home automation (unlimited switches control)

e. Long distance Data transmission.

f. Military communication through encrypted data of DTMF values.

\section{ACKNOWLEDGMENTS}

Our thanks to the experts who have contributed towards development of the template.

\section{CONCLUSION}

DTMF is a reliable technique for very long distance data transmission. Though Genave Super Fast rate of 20/20 (25 tunes per second) from an automatic encoder or Genave decoder responding to a code sent at a blazing DTMF rate of 20/5 also known as 40 digits per second)[17]. If the bits rate can be increased then DTMF will become a good communication way for short distance also. But for long distance and unlimited node wireless sensor network it is very reliable, easy, cheap solution for small amount of data transmission.

\section{REFERENCES}

[1] Corke, P., Wark, T., Jurdak, R., Hu, W., Valencia, P. and Moore, D., "Environmental Wireless Sensor Networks", IEEE Sensors, Vol. 98, No. 11, 2010, pp. 1903-1917.

[2] Vana, Tomislav, Dinko, Marijan kuri and Vendran Bilas.," MasliNET: A Wireless Sensor Network based Environmental Monitoring System", MIPRO 2011, May 23-27, 2011, Opatija, Croatia.

[3] Garcia-Romeo, D; Fuentes, H. ; Medrano, N. ; Calvo, B." A NDIR-based $\mathrm{CO} 2$ monitor system for wireless sensor networks", Circuits and Systems (LASCAS), 2012 IEEE Third Latin American Symposium on, Feb. 29 2012-March 22012.
[4] Bencini, L., Di Palma, D., Collodi, G, Manes, G. and Manes, A.,"Wireless Sensor Networks for On-field Agricultural, Management Process", Wireless Sensor z

[5] Yun Chan Cho and Jae wook Jeon,IEEE International conference on Industrial Informatics (INDIN 2008) DCC, Daejeon, Korea, July 13-16,2008, pp. 1441-1446.

[6] D. Manojkumar, P. Mathankumar, trolled Robot using DTMF Engineering Research, 2010, 2(3), PP. 349-355.

[7] Tulijappa M Ladwa, Sanjay M Ladwa, R Sudhrashan Kaarthik, Alok Ranjan Dhara and Nayan Dalei, Communications, Information Technology, and Biomedical Engineering (ICICI-BME), Bandung, Indonesia, 2009, pp. 1-6.

[8] Daniel H International Workshop on Intelligent Data Acquisition and Advanced Computing Systems:Technology and Applications, Rende (Cosenza), Italy 21-23 September 2009, pp.625-628

[9] Ali Sekman, Ahmet Bugra Koku, and Saleh ZeinSabatto, IEEE International Conference on Systems, Man and Cybernetics, 2003, 4,PP.3937-3942

[10] T. Kubik and M. Sugisaka, "Use of a Cellular Phone in Mobile Robot Voice Control", Proceedings of the $40^{\text {th }}$ SICE Annual Conference. International Session Papers, Naogoya, 2001, pp.106-111.

[11] Tho Nguyen and Linda G. Bushnell, "Feasibility Study of DTMF Communications for Robots", UWEE Technical Report Number [TRS Number],April 6, 2004.

[12] http://dx.com/p/i2c-rtc-ds1307-24c32-real-time-clockmodule-for-arduino-blue-149493 for data about real time clock module.

[15] Atmega8 datasheet.

[16] http://www.arduino.cc/en/Main/ArduinoBoardUno for data about arduino Uno.

[17] http://www.genave.com/dtmf-encoders-manualautomatic. htm for latest DTMF transfer rate. 